\title{
FORNECIMENTO DE VOLUMOSO PARA BEZERROS PRÉ-RUMINANTES
}

\author{
ROUGHAGE FOR PRE-RUMINANT CALVES
}

\section{Rosane Scatamburlo Lizieire ${ }^{1}$ Daniel de Noronha Figueiredo Vieira da Cunha ${ }^{2}$ Janaína Azevedo Martuscello ${ }^{2}$ Oriel Fajardo de Campos ${ }^{3}$}

\section{RESUMO}

$O$ presente trabalho avaliou a performance de bezerros mestiços HolandêsxZebu, do nascimento aos 90 dias de idade, desaleitados aos 56 dias, com acesso ou não a volumoso. O ensaio foi conduzido na Estação Experimental de Itaguaí da PESAGRO-RIO, em Seropédica, RJ, no período de janeiro a abril de 2000. Foram utilizados 27 bezerros, distribuídos em blocos ao acaso, de acordo com o peso ao nascer e com o sexo, nos seguintes tratamentos experimentais: (1) Concentrado; (2) Concentrado + pasto de capim-estrela (Cynodon nhenfluensis), $e$ (3) Concentrado+feno de alfafa (Medicago sativa). Os animais foram separados das mães 24 horas após o nascimento $e$ mantidos em baias individuais. Todos os animais receberam colostro até o $3^{\circ}$ dia de vida e, a partir daí, quatro litros de leite integral/animal/dia, além de concentrado comercial (18\%PB), até o máximo de 2,0kg/animal/dia, a partir da segunda semana de vida. Os animais do tratamento 2 tiveram acesso aos piquetes, manejados em rodízio, a partir do $15^{\circ}$ dia de vida, no período das 8 às $14 h$, e aqueles do tratamento 3 receberam feno de alfafa $(18 \% \mathrm{~PB})$ picado, à vontade, a partir do $8^{\circ}$ dia de vida. Não houve diferença $(P>0,05)$ entre os tratamentos 1 e 3; entretanto, aqueles submetidos ao tratamento 2 consumiram menor $(P<0,05)$ quantidade de concentrado. Não houve diferença $(P>0,05)$ para consumo total de matéria seca e eficiência alimentar entre os tratamentos 1 e 3 e para ganho de peso médio diário, perímetros abdominal e torácico, incidência de diarréias e corrimento nasal entre os três tratamentos. O fornecimento de volumoso para bezerros pode ser feito a partir da $8^{a}$ semana de idade, sem prejuízos para o seu desenvolvimento, desde que os animais tenham à sua disposição concentrado inicial desde a segunda semana de idade.

Palavras-chave: bezerros, bovinos de leite, volumoso, desaleitamento precoce.

\section{SUMMARY}

This experiment aimed to evaluate the growth of Holstein Zebu crossbred calves, from birth to 90 days of age, early weaned, with or without available roughage from the second week of age on. The trial was carried out at the Itaguaí Experimental Station, from PESAGRO-RJ, from January up to April, 2000. Twenty-seven calves were used, randomly assigned to blocks according to sex and birth weight, in the following experimental treatments: (1) only starter; (2) starter plus Cynodon nhenfluensis paddocks grazed from the $15^{\text {th }}$ day of age on; and, (3) starter plus Medicago sativa hay. Calves were separated from their dams twenty-four hours after birth and kept to individual pens. All animals received colostrum up to the $3^{\text {rd }}$ day of age and, from there on, four litters of whole milk/animal/day, up to the $56^{\text {th }}$ day of age, and commercial starter $(18 \% \mathrm{CP})$ up to a maximum of $2.0 \mathrm{~kg} /$ animal/day from the second week of age on. Animals from treatments 1 and 3 were kept on their pens up to 70 days of age, those from treatment 3 received, in separated feeders, chopped alfalfa hay $(18 \% \mathrm{CP}$, particle size of $5 \mathrm{~cm})$, ad libitum, from the $8^{\text {th }}$ day on. Animals in treatment 2 had access to paddocks from 8:00 to 14: 00h. Milk, starter and hay intakes, as well as diarrhoea and nasal flow incidences were measured daily up to 70 days of age. Body weight and barrel and heart girth were taken weekly. There were no difference $(P>0.05)$ between calves fed roughage (treatments 2 and 3) for starter intake; however, animals allowed to graze showed lower $(P<0.05)$ starter intake. Average total dry matter intake and feed efficiency were similar $(P>0.05)$ for treatments 1 and 3. There were no differences $(P>0.05)$ among treatments for average daily weight gain, as well as diarrhea, nasal flow incidences and barrel and heart girth. It was concluded that roughage may be supplied to calves from day $56^{\text {th }}$ of age on, without impairing their development, when starter is offered since the second week of age.

Key words: calves, dairy calves, roughages, early weaning.

\footnotetext{
${ }^{1}$ Zootecnista, MSc., Estação Experimental de Itaguaí, PESAGRO, RJ, BR 465, km 47, 23836-710, Seropédica, RJ. Telefax: (021)26821074. E-mail:eei@domain.com.br. Autor para correspondência.

${ }^{2}$ Estudantes do Instituto de Zootecnia da Universidade Federal Rural do Rio de Janeiro, BR 465, km 47, 23.836-710, Seropédica, RJ.

${ }^{3}$ Zootecnista, PhD., Embrapa Gado de Leite, Rua Eugênio do Nascimento, 610, 36038-330, Juiz de Fora, MG.
} 


\section{INTRODUÇÃO}

Quando se adota o desaleitamento precoce, dois aspectos devem ser levados em consideração: o custo e o desenvolvimento do bezerro. O primeiro é, sem dúvida, o principal objetivo do desaleitamento precoce, pois quanto mais cedo o bezerro for desaleitado, mais leite poderá ser comercializado. Por outro lado, se o bezerro não estiver com o seu aparelho digestivo completamente desenvolvido, para o aproveitamento de alimentos sólidos, o lucro proveniente da venda do leite, que deveria ter sido fornecido ao bezerro, poderá ser anulado com o aumento dos índices de morbidade e mortalidade. $\mathrm{O}$ fornecimento de volumoso e concentrado para bezerros préruminantes tem sido adotado para promover o seu desenvolvimento e permitir o corte no fornecimento da dieta líquida o mais cedo possível (QUIGLEY, 1998). O alimento sólido, seja concentrado, feno ou ambos, resulta em aumentos marcantes no rúmenretículo e omaso (WARNER et al., 1956). O desenvolvimento em termos de volume pode ser conseguido somente com alimento volumoso e até com material inerte (maravalha, serragem ou esponjas), apesar do menor crescimento do bezerro (STOBO et al., 1966); já o desenvolvimento fisiológico está associado aos ácidos graxos voláteis (AGV), normalmente absorvidos pelas paredes do rúmen, e ao desenvolvimento das papilas (ANDERSON $\boldsymbol{e t} \boldsymbol{a} \boldsymbol{l} ., \quad$ 1987, ASSANE \& DARDILLAT, 1994). Entretanto, existem algumas contradições em relação ao fornecimento de volumoso para bezerros pré-ruminantes. DREVJANY (1986) não recomenda o fornecimento de feno para bezerros de rebanhos leiteiros aproveitados para a produção de carne, e QUIGLEY (1998) comenta que o feno deve ser parte da dieta dos bezerros somente após o desaleitamento, uma vez que, antes disso, o consumo é muito baixo e a exigência em energia dos animais pode ser mantida com a dieta líquida e o concentrado.

O presente trabalho teve por objetivo avaliar o desenvolvimento de bezerros mestiços Holandês x Zebu submetidos a três estratégias de alimentação, com o fornecimento de concentrado e/ou volumoso a partir da segunda semana de idade, desaleitados precocemente.

\section{MATERIAL E MÉTODOS}

O experimento foi conduzido na Estação Experimental de Itaguaí, da PESAGRO-RJ, localizada no km 47 da Estrada Rio-São Paulo, em Seropédica, RJ. Foram utilizados 27 bezerros, mestiços Holandês-Zebu, do nascimento aos 90 dias de idade. Os animais foram distribuídos em blocos ao acaso, de acordo com o peso ao nascer e com o sexo, nos seguintes tratamentos experimentais: (1) Concentrado; (2) Concentrado+pasto de capimestrela (Cynodon nhenfluensis); e (3) Concentrado+feno de alfafa (Medicago sativa). Os animais foram mantidos em baias individuais, de alvenaria, dotadas de bebedouro, cochos para concentrado e feno, até os 70 dias de idade, e aleitados, artificialmente, em baldes. Todos os animais receberam concentrado comercial peletizado (18\%PB) a partir da segunda semana de idade, limitando-se o consumo em $2 \mathrm{~kg} / \mathrm{animal} / \mathrm{dia}$. Os animais do tratamento 2 tinham acesso aos piquetes, manejados em rodízio, a partir do $15^{\circ}$ dia de vida, no período das $8 \mathrm{~h}$ às $14 \mathrm{~h}$ do dia, quando retornavam às respectivas baias. Os animais do tratamento 3 , por sua vez, receberam feno de alfafa $(18 \% \mathrm{~PB})$ picado $(5 \mathrm{~cm})$ em cochos separados, à vontade, a partir do $8^{\circ}$ dia de vida. Os bezerros foram apartados das mães 24 horas após o nascimento e receberam quatro litros de colostro/animal/dia, em baldes, até o $3^{0}$ dia de vida. A partir daí, receberam quatro litros de leite integral/animal/dia, divididos em duas refeições diárias até o $10^{\circ}$ dia de vida, e somente à tarde, do $11^{\circ}$ dia até o desaleitamento. $\mathrm{O}$ corte no fornecimento da dieta líquida foi realizado no $57^{\circ} \stackrel{\text { }}{ }$ dia de idade, abruptamente. Após o desaleitamento, os animais permaneceram na mesma instalação até os 70 dias de idade, quando, então, foram transferidos para os piquetes de capim-estrela, onde permaneceram até o final do experimento. Foram realizadas as seguintes mensurações: consumo diário de concentrado e feno, consumo diário de dieta líquida, pesagens semanais, perímetro torácico e abdominal tomados semanalmente, incidência diária de diarréias e de corrimento nasal. Os dados de desempenho foram submetidos à análise da variância. As médias dos fatores quantitativos foram comparadas pelo teste de Tuckey, adotando-se o nível de 5\% de probabilidade. As análises estatísticas foram realizadas utilizando-se o Sistema de Análises Estatísticas Genéticas-SAEG (EUCLIDES, 1990). Os dados relacionados à incidência de diarréias e ao corrimento nasal foram analisados pelo Teste do Qui-quadrado.

\section{RESULTADOS E DISCUSSÃO}

Os dados referentes não só ao consumo de concentrado e ganho médio de peso diário, nos

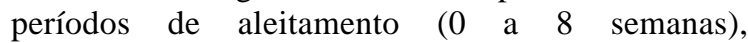
imediatamente após o desaleitamento (9-10 semanas) e no período total (0-10 semanas), como também ao peso vivo ao nascimento, ao desaleitamento (56 dias) e aos noventa dias de idade são mostrados na tabela 1 . No período de 
Tabela 1 - Peso ao nascimento, ao desaleitamento (56 dias) e aos 90 dias de idade, consumo de concentrado e ganho médio de peso diário dos bezerros submetidos aos diferentes tratamentos experimentais.

\begin{tabular}{|c|c|c|c|c|}
\hline & Concentrado & $\begin{array}{l}\text { Concentrado } \\
+ \text { Pasto }\end{array}$ & $\begin{array}{l}\text { Concentrado } \\
+ \text { Feno }\end{array}$ & $\mathrm{EP}^{1}$ \\
\hline Peso ao nascer, $\mathrm{kg}$ & $31,8^{\mathrm{a}}$ & $31,4^{\mathrm{a}}$ & $31,8^{\mathrm{a}}$ & 0,43 \\
\hline Peso vivo aos 56 dias, $\mathrm{kg}$ & $58^{\mathrm{a}}$ & $57^{\mathrm{a}}$ & $59^{\mathrm{a}}$ & 1,9 \\
\hline Peso vivo aos 90 dias, $\mathrm{kg}$ & $79^{a}$ & $76^{\mathrm{a}}$ & $80^{\mathrm{a}}$ & 2,9 \\
\hline \multicolumn{5}{|c|}{ Consumo de concentrado, g/an/dia } \\
\hline 0 a 8 semanas & $347^{\mathrm{a}}$ & $227^{\mathrm{b}}$ & $358^{\mathrm{a}}$ & 42 \\
\hline 9 a 10 semanas & $1.297^{\mathrm{a}}$ & $875^{\mathrm{b}}$ & $1.124^{\mathrm{ab}}$ & 102 \\
\hline 0 a 10 semanas & $519^{\mathrm{a}}$ & $346^{\mathrm{b}}$ & $489^{\mathrm{ab}}$ & 47 \\
\hline \multicolumn{5}{|c|}{ Ganho de peso médio diário, g/animal/dia } \\
\hline 0 a 8 semanas & $439^{\mathrm{a}}$ & $427^{\mathrm{a}}$ & $452^{\mathrm{a}}$ & 30 \\
\hline 9 a 10 semanas & $521^{a}$ & $439^{a}$ & $513^{\mathrm{a}}$ & 47 \\
\hline 0 a 10 semanas & $533^{a}$ & $498^{\mathrm{a}}$ & $541^{\mathrm{a}}$ & 35 \\
\hline 0 a 13 semanas & $517^{\mathrm{a}}$ & $432^{a}$ & $534^{\mathrm{a}}$ & 31 \\
\hline
\end{tabular}

${ }^{\mathrm{a}, \mathrm{b}}$ Médias, na mesma linha, seguidas de letras diferentes, diferem entre si em nível de 5\% de probabilidade pelo Teste de Tukey.

aleitamento, os animais do tratamento 2 consumiram significativamente menor $(\mathrm{P}<0,05)$ quantidade de concentrado quando comparados àqueles dos tratamentos 1 e 3, sendo que estes dois últimos não diferiram $(\mathrm{P}>0,05)$ entre si. COELHO (1999) não encontrou diferenças significativas para consumo e ganho de peso de bezerros desaleitados aos trinta dias de idade que receberam dietas com ou sem a participação do feno de Tifton 85. Já CASTRO \& ZANETTI (1998) obtiveram consumos de matéria seca significativamente maiores para bezerros que receberam feno de capim-coastcross (Cynodon dactylon (1.) Pers) picado, incorporado ou fornecido separadamente, comparados àqueles que receberam somente concentrado. Os valores médios para consumo de concentrado, obtidos neste ensaio, são semelhantes àqueles reportados na literatura (COELHO, 1999, GREENWOOD et al., 1997, McGAVIN \& MORRIL, 1976) para bezerros até seis a oito semanas de idade (variando de 295 a 489 g por dia). Vale ressaltar que, nessa fase, o consumo de concentrado é relativamente baixo porque a dieta líquida é a principal fonte de nutrientes e, neste experimento, todos animais receberam a mesma quantidade de leite integral.

Logo após o desaleitamento $\left(\begin{array}{lll}9 & \text { a } & 10\end{array}\right.$ semanas), período considerado crítico, e de 0 a 10 semanas de idade, os animais que tinham acesso ao pasto a partir do $15^{\circ}$ dia de vida também consumiram menor $(\mathrm{P}<0,05)$ quantidade de concentrado em relação aos animais do tratamento 1 (concentrado), mas não diferiram $(\mathrm{P}>0,05)$ daqueles alimentados com feno (tratamento 3). O menor consumo de concentrado obtido pelos animais do tratamento 2, provavelmente, se deveu ao fato de esses bezerros permanecerem parte do dia nos piquetes, tendo menor tempo disponível para consumir o concentrado. Em conseqüência, o consumo de concentrado dos animais do tratamento 2 , no momento do desaleitamento, às oito semanas, ficou abaixo do consumo mínimo (600 a 700g por animal por dia) recomendado pela literatura (CAMPOS \& LIZIEIRE, 1998). Entretanto, este limite parece não ter sido suficientemente baixo para influenciar o ganho de peso dos animais neste ensaio (Tabela 1). Provavelmente, o pasto tenha contribuído para minimizar essa diferença. Já os animais que receberam concentrado (tratamentos 1) ou concentrado e feno de alfafa (tratamento 3) apresentaram consumos de concentrado superiores a 600700g/animal/dia (Figura 1).

Não houve diferença $(\mathrm{P}>0,05)$ entre tratamentos para os ganhos de peso médios diários nos três períodos considerados, apesar de os animais que tiveram acesso ao pasto, a partir do $15^{\circ}$ dia de vida, terem consumido menos $(\mathrm{P}<0,05)$. Contrativamente, CASTRO \& ZANETTI (1998) mostraram que o fornecimento de feno de capim-coastcross (Cynodon dactylon (1.) Pers), picado e incorporado na dieta ou fornecido separado e à vontade, aumentou significativamente o ganho de peso dos animais quando comparados àqueles que receberam somente concentrado, embora a conversão alimentar não tenha sido diferente entre os tratamentos. Os ganhos médios de peso diário obtidos neste ensaio foram semelhantes àqueles obtidos por outros autores (QUIGLEY et al., 1991, LUCCI, 1976, PLAZA et al., 1990) para animais até 13 semanas de idade. Um dado importante a ser ressaltado é que, independente dos tratamentos, os animais não perderam peso logo após o desaleitamento (9-10 semanas), período considerado crítico devido à retirada da dieta líquida, quando, normalmente, ocorrem as maiores perdas de peso (Figura 2). Conseqüentemente, não houve diferença significativa $(\mathrm{P}>0,05)$ entre os tratamentos estudados para peso ao desaleitamento e aos 90 dias de idade, estando esses valores próximos daqueles recomendados para animais mestiços Holandês $\mathrm{x}$ Zebu (CAMPOS \& LIZIEIRE, 2000). Não houve diferença $(\mathrm{P}>0,05)$ para o consumo total de matéria seca e a eficiência alimentar entre os animais que receberam concentrado e concentrado + feno (Tabela 2). Como o consumo de pasto não foi estimado, o tratamento 2 não foi considerado nesta comparação. Os consumos totais de matéria seca 


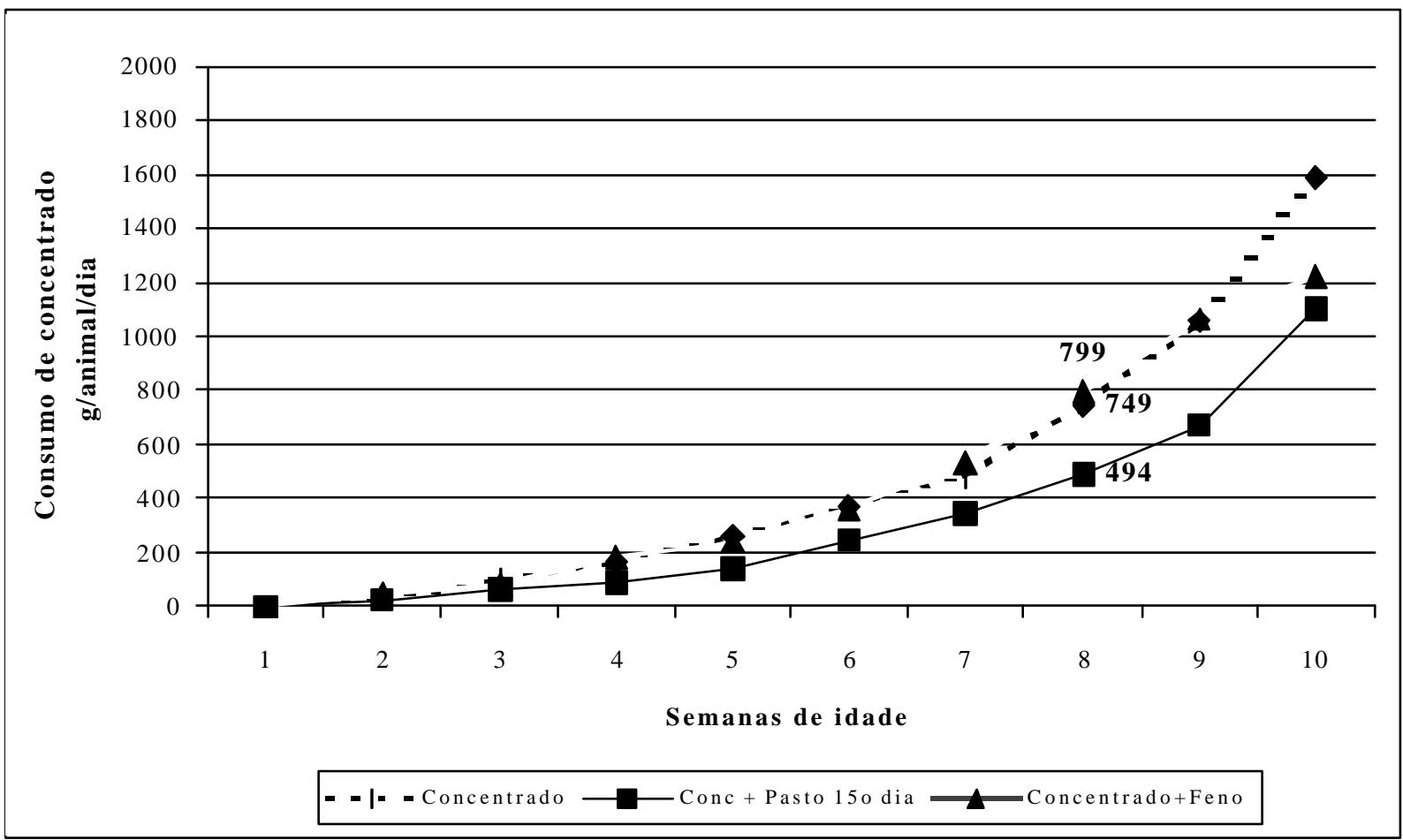

Figura 1 - Consumo médio de concentrado pelos animais, em g/animal/dia, do nascimento às dez semanas de idade.

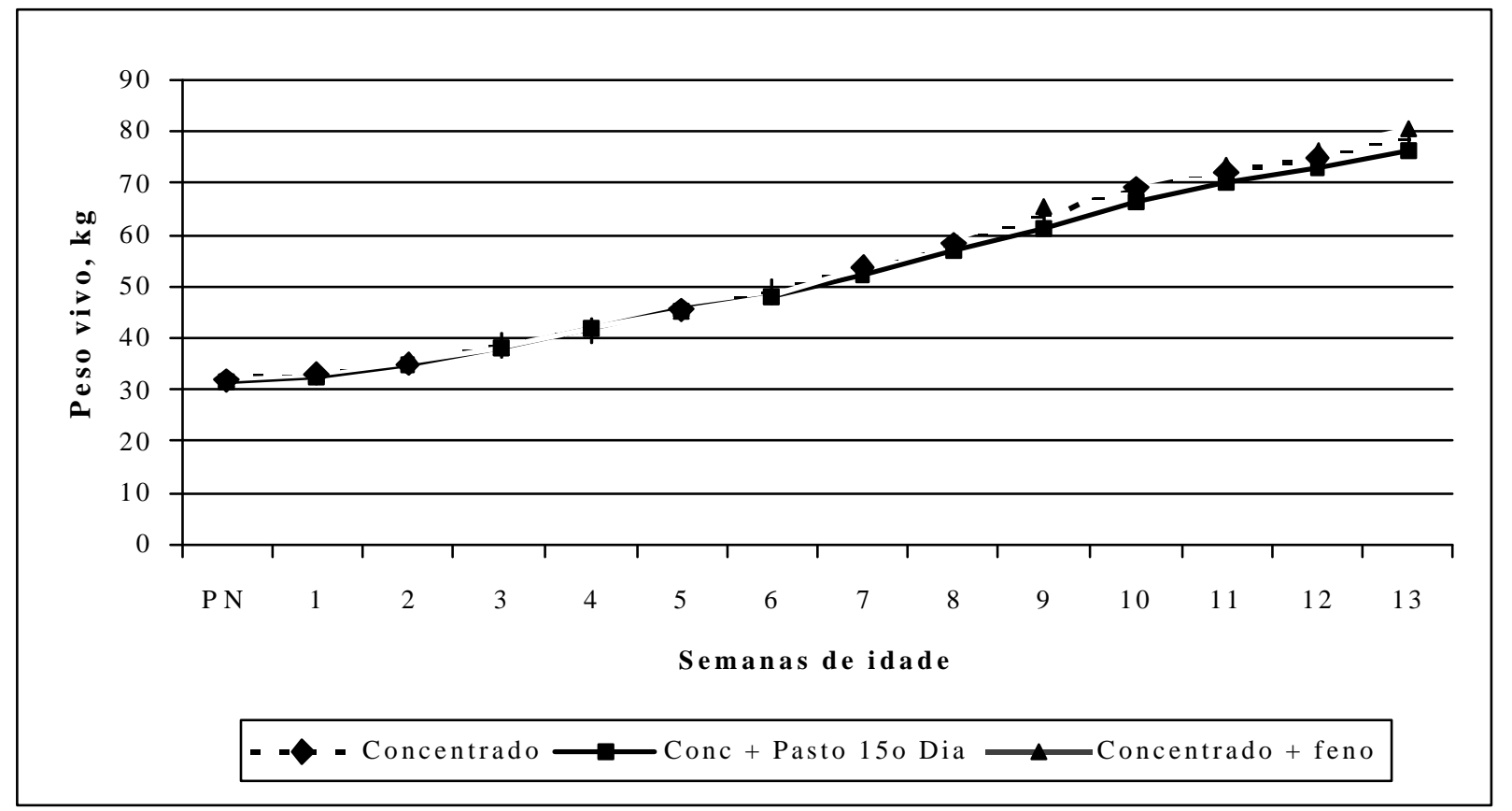

Figura 2 - Peso vivo médio dos animais, do nascimento às treze semanas de idade.

obtidos neste ensaio são semelhantes àqueles encontrados por CASTRO \& ZANETTI (1998) para bezerros que receberam feno de capim-coastcross (Cynodon dactylon (1.) Pers), incorporado ou não na dieta dos animais. Além disso, os animais que consumiram feno não apresentaram $(\mathrm{P}>0,05)$ abdômen mais distendido daqueles que não receberam, conforme mencionado por DREVJANY

Ciência Rural, v. 32, n. 5, 2002. 
Tabela 2 - Consumo total de matéria seca (g/animal/dia) e eficiência alimentar ( $\mathrm{kg}$ de ganho/kg de matéria seca consumida ) dos animais submetidos ao tratamento 1 (concentrado) e ao tratamento 3 (concentrado + feno).

\begin{tabular}{lccc}
\hline & Concentrado & $\begin{array}{c}\text { Concentrado } \\
+ \text { Feno }\end{array}$ & EP $^{1}$ \\
\hline \multicolumn{4}{c}{ Consumo total de matéria seca, g/an/dia } \\
0 a 8 semanas, & 826 & 854 & 42 \\
9 a 10 semanas & 1.297 & 1.317 & 89 \\
0 a 10 semanas & 999 & 993 & 48 \\
& Eficiência alimentar & \\
0 a 8 semanas & 0,53 & 0,54 & 0,03 \\
9 a 10 semanas & 0,40 & 0,40 & 0,04 \\
0 a 10 semanas & 0,54 & 0,55 & 0,02 \\
\hline
\end{tabular}

${ }^{1} \mathrm{EP}=$ Erro padrão da média

${ }^{\mathrm{a}, \mathrm{b}}$ Médias, na mesma linha, seguidas de letras diferentes, diferem entre si ao nível de 5\% de probabilidade pelo Teste de Tukey.

(1986); talvez em função do baixo consumo de feno pelos bezerros.

Os consumos médios de feno foram 16 , 60 e $24 \mathrm{~g}$ por animal por dia, respectivamente, nos períodos de 0-8, 9-10 e 0-10 semanas de idade, aumentando somente a partir da $7^{\mathrm{a}}$ semana de idade (Figura 3). Esses resultados estão próximos daqueles obtidos por outros autores (QUIGLEY et al., $1985 \mathrm{e}$ COELHO, 1999) e reforça a opinião de QUIGLEY (1998), de que somente após as seis semanas de idade o feno constitui parte importante da dieta desses animais. Segundo este autor, antes das seis semanas de idade, os animais não consomem quantidades significativas de feno se o concentrado também estiver disponível e, na maioria das vezes, o feno acaba sendo utilizado como cama para os animais. Mesmo que o volumoso seja de excelente qualidade, não daria suporte para as exigências nutricionais de bezerros pré-ruminantes, principalmente em termos de energia, que, nessa fase, é fornecida, principalmente, pela dieta líquida e pelo concentrado. Segundo PLAZA et al. (1990), o fornecimento do feno separado do concentrado pode ser uma das razões para o seu baixo consumo pelos bezerros. Vale ressaltar ainda que, com exceção de PLAZA et al. (1990), que utilizaram feno de capimcoastcross (Cynodon dactylon (1.) Pers), todos os outros autores utilizaram feno de alfafa.

A análise pelo teste do Qui-quadrado mostrou que a ocorrência de diarréias foi dependente do tratamento experimental. Os bezerros que receberam feno ou pasto a partir do $15^{\circ}$ dia de idade apresentaram maior $(\mathrm{P}>0,05)$ número de dias com diarréia do que aqueles que não receberam volumoso. Vale ressaltar, contudo, que esta diferença foi devida a apenas dois bezerros, um no tratamento com acesso a pasto e outro no tratamento com feno. Portanto, há que se questionar se de fato os alimentos volumosos foram os responsáveis

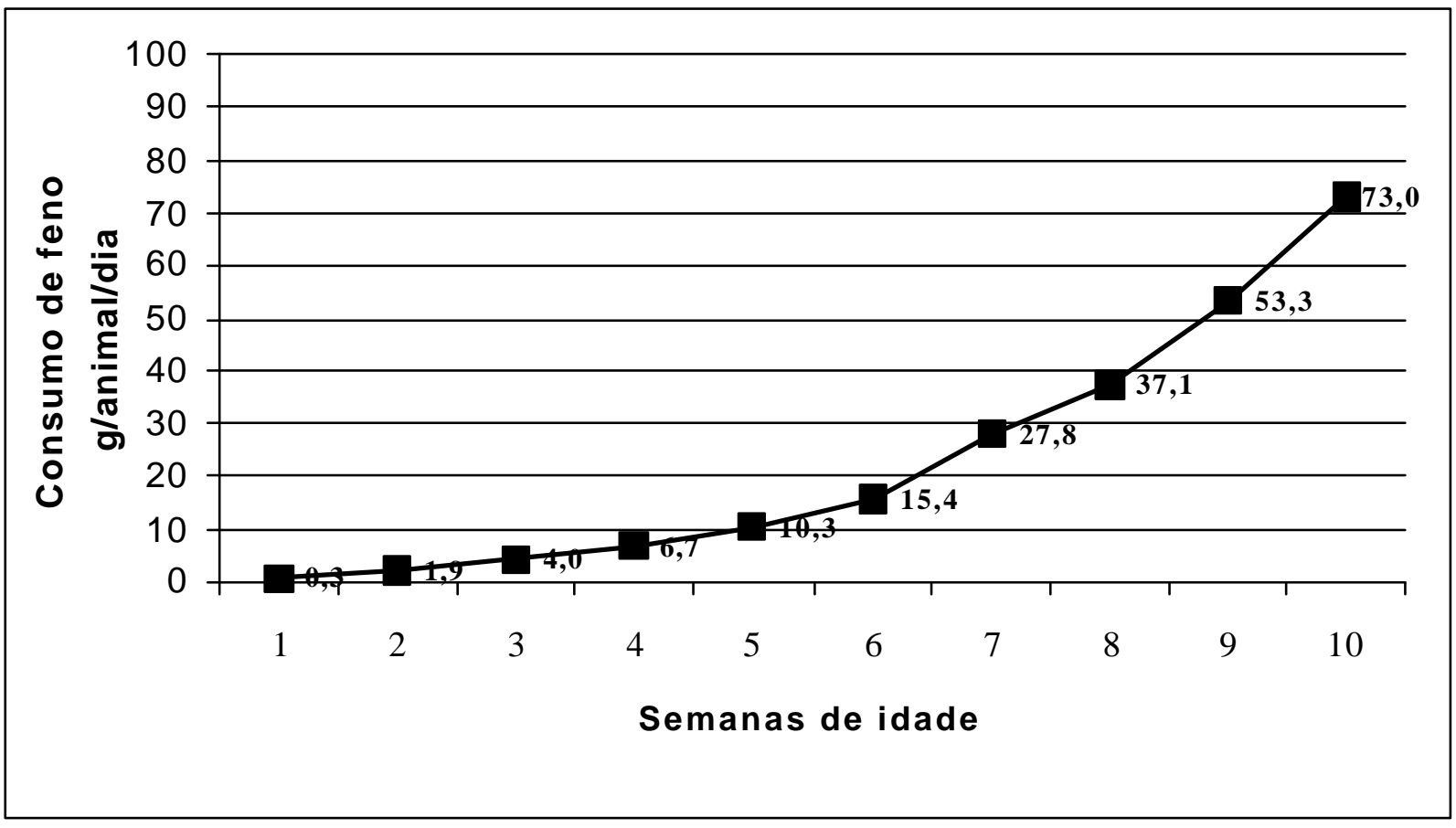

Figura 3 - Consumo de feno de alfafa (Medicago sativa), a partir da segunda semana de idade.

Ciência Rural, v. 32, n. 5, 2002. 
pelas diarréias observadas. A incidência de corrimento nasal também foi insignificante $\mathrm{e}$ semelhante $(\mathrm{P}>0,05)$ para os três tratamentos experimentais. Embora não tenha sido avaliado, talvez a grande vantagem do tratamento 2 (pasto a partir do $15^{\circ}$ dia de vida) tenha sido proporcionar aos animais condições de menor estresse, uma vez que passavam parte do dia em piquetes e não confinados em baias, como os demais tratamentos

\section{CONCLUSÕES}

Em sistemas de aleitamento artificial, com desaleitamento aos 56 dias de idade, o fornecimento de volumoso pode ser feito a partir da $8^{\text {a }}$ semana de idade, desde que os animais tenham à sua disposição concentrado inicial, desde a segunda semana de idade.

\section{REFERÊNCIAS BIBLIOGRÁFICAS}

ANDERSON, K.K., NAGARAJA, T.G., MORRILL, J.L. Ruminal metabolic development in calves weaned conventionally or early. Journal of Dairy Science. Champaign, v.70, n.5, p.1000-1005, 1987.

ASSANE, M., DARDILLAT, C. Influence d' une supplémentation solide sur la physiopathologie digestive du veau préruminant. Revue Du Medicine Vétérinaire., Toulouse, v.145, n.6, p.461-469, 1994.

CAMPOS, O.F., LIZIEIRE, R.S. Estratégias para obtenção de fêmeas de reposição em rebanhos leiteiros. In: SIMPÓSIO SOBRE PRODUÇÃO ANIMAL, 10., 1998, Piracicaba, SP. Anais... Piracicaba : FEALQ, 1998. p.215-255.

CAMPOS, O.F., LIZIEIRE, R.S. Desaleitamento precoce e alimentação de bezerras. In: SIMPÓSIO SOBRE MANEJO E NUTRIÇÃO DE GADO DE LEITE, 2000, Goiânia, GO. Anais... Goiânia : Colégio Brasileiro de Nutrição Animal, 2000. p.1-20.

CASTRO, A.K.M., ZANETTI, M.A. Estudo da inclusão de fibra na dieta de bezerros da raça holandesa. Revista da Sociedade Brasileira Zootecnia., Viçosa, v.27, n.6, p.1193-1198, 1998.

COELHO, S.G. Ganho de peso e desenvolvimento do estômago de bezerros desleitados aos trinta dias de idade e alimentados com concentrado e com ou sem feno. Belo
Horizonte, 1999. 123p. Tese (Doutorado em Nutrição Animal) Universidade Federal de Minas Gerais, Belo Horizonte, 1999.

DREVJANY, L.A. Towards success in heavy calf production. Kemptville : Ontario Ministry of Agriculture and Food, 1986. $54 \mathrm{p}$.

EUCLIDES, R.F. Sistema de análise estatística genética Viçosa : UFV, 1990. 68p.

GREENWOOD, R.H., MORRIL, J.L., TITGEMEYER, E.C. $\boldsymbol{e}$ $a l$. A new method of measuring of diet abrasion and its effects on the development of the forestomach. Journal of Dairy Science, Champaign, v.80, n.10, p.2534-2541, 1997.

LUCCI, C.S. Desaleitamento precoce de bezerros. III. Desenvolvimento do rúmen. Revista da Faculdade de Medicina Veterinária e Zootecnia da USP, São Paulo, v.13, n.2, p.333-338, 1976.

McGAVIN, M.D., MORRILL, J.L. Scanning electron microscopy of ruminal papillae in calves fed various amounts and forms of roughage American Journal of Veterinary Research, Schaumburg, v.37, n.5, p.497-508, 1976.

PLAZA, J., FERNANDEZ, E., MERINO, N. et al. The effect of the level of ground hay in starter concentrates on calf performance. Cuban Journal of Agricultural Science, v.24, n.1-3, p.291-297, 1990

QUIGLEY, J.D. III, SCHWAB, C.G., HYLTON, W.E. Development of rumen function in calves: nature of protein resting the abomasum. Journal of Dairy Science, v.68, n.13, p.694-702, 1985 .

QUIGLEY, J.D. III, CALDWELL, L.A., SINKS, G.D. et al. Changes in blood glucose, nonesterified fatty acids, and ketones in response to weaning feed intake in young calves. Journal of Dairy Science, Champaign, v.74, n.1, p.258-263, 1991.

QUIGLEY, J.D. III. Does hay develop the rumen? (01 Feb. 98) APCcalf notes.http://www.americanprotein.com. Capturado em 27 de março de 1998.

STOBO, I.J.F., ROY, J.H.B., GASTON, H.J. Rumen development in the calf. I. The effect of diets containing different proportions of concentrates to hay on rumen development. British of Journal of Nutrition, London, v.20, n.2, p.171-188, 1966.

WARNER, R.G., FLATT, W.P., LOOSLI, J.K. Dietary factors influencing the development of the ruminant stomach. Journal of Agricultural Chemistry, Washington, v.4, p.788-792, 1956 